\title{
Partial ex-post verifiability and unique implementation of social choice functions
}

\author{
Hitoshi Matsushima ${ }^{1}$ (D)
}

Received: 25 February 2019 / Accepted: 21 September 2020 / Published online: 8 October 2020

(C) The Author(s) 2020

\begin{abstract}
This study investigates the unique implementation of a social choice function in iterative dominance in the ex-post term. We assume partial ex-post verifiability; that is, after determining an allocation, the central planner can observe partial information about the state as verifiable. We demonstrate a condition of the state space, termed "full detection," and show that with full detection, any social choice function is uniquely implementable even if the information that can be verified ex-post is very limited. To prove this, we construct a dynamic mechanism according to which each player announces his (or her) private signal, before the other players observe this signal, at an earlier stage, and each player also announces the state at a later stage. In this construction, we can impose several severe restrictions such as boundedness, permission of only tiny transfers off the equilibrium path, and no permission of transfers on the equilibrium path. This study does not assume either expected utility or quasi-linearity.
\end{abstract}

\section{Introduction}

This study investigates the unique implementation of a social choice function (SCF). The equilibrium concept we adopt is an iteratively undominated strategy in the ex-post term, which is a very weak notion. Therefore, the uniqueness requirement is demanding. To achieve the allocation implied by a social choice function, which is contingent on the state, the unaware central planner must require informed players to reveal what they know about the state. Hence, the central planner must construct

\footnotetext{
This is a drastic revision of a part of Matsushima (2017). This study was supported by a Grantin-aid for scientific research (KAKENHI 25285059) from the Japan Society for the Promotion of Science (JSPS) and the Ministry of Education, Culture, Sports, Science and Technology (MEXT) of the Japanese government. I am grateful to Takashi Kunimoto, Shunya Noda, Rene Saran, Satoru Takahashi, and Takashi Ui for their useful comments. All errors are mine.

Hitoshi Matsushima

hitoshi@e.u-tokyo.ac.jp

1 Department of Economics, University of Tokyo, Hongo, Bunkyo-ku, Tokyo 113-0033, Japan
} 
an appropriate mechanism that incentivizes each player to make the desirable (i.e., truthful) announcements as unique equilibrium behavior. This construction should be generally regarded as a difficult task.

To overcome the difficulty, we assume partial ex-post verifiability, that is, after determining an allocation, the central planner can only observe partial information about the state. This observation is verifiable and contractible ex-post. Hence, to resolve the difficulty presented by this uniqueness issue, the central planner makes ex-post monetary transfers with these players. These transfers are contingent not only on players' announcements, but also on the verifiable information. We thus present the possibility that this partial ex-post verifiability fully solves the unique implementation problem.

We assume complete information about the state just before determining an allocation. We further assume incomplete information such that each player observes his (or her) private signal concerning the state earlier than the other players do. We design a dynamic mechanism, such that the central planner requires each player to announce his (or her) private signal at the earlier stage and announce the whole description of the state at the later stage.

Based on this dynamic procedure of information acquisition and revelation, we introduce a condition on the state space, which we term full detection. We show that with full detection, an SCF is uniquely implementable via iterative dominance even if the information that can be verified ex-post is very limited, that is, even if the range of the players' lies that the ex-post verifiable information can directly detect is quite narrow.

Full detection requires the ex-post verifiable information to detect only a limited class of players' lies. However, the elimination of these detected lies can help in turn detect another class of lies. By using this "chain of detection," we can iteratively detect all possible lies.

We show that full detection is a necessary condition for the existence of a direct revelation mechanism in which truth-telling is the unique iteratively undominated strategy for any player who concerns only his (or her) monetary transfer. We then show our main theorem, stating that full detection is generally sufficient for unique implementation via iterative dominance.

The dynamic mechanism in our main theorem satisfies various severe restrictions such as boundedness (e.g., Jackson 1992), permission of only tiny monetary transfers off the equilibrium path, and no permission of transfers on the equilibrium path. Importantly, we do not assume either expected utility or quasi-linearity. In fact, we only make basic assumptions on preferences, such that each player's utility function is continuous in lottery over allocations and is continuous and increasing in monetary transfers.

The literature on implementation already establishes that it is generally impossible to uniquely (or fully) implement an SCF in a Nash equilibrium if there is no such verifiable signal. ${ }^{1}$ For instance, Makin-monotonicity is a necessary

\footnotetext{
${ }^{1}$ For surveys on implementation theory, see Moore (1992), Palfrey (1992), Osborne and Rubinstein (1994, Chapter 10), Jackson (2001), and Maskin and Sjöström (2002).
} 
condition for an SCF to be implementable in Nash equilibrium (e.g., Maskin 1999). However, Maskin-monotonicity is quite a demanding condition for a deterministic SCF.

Several works attempt to weaken the requirements of unique implementation to derive the corresponding possibility results. For instance, Matsushima (1988) and Abreu and Sen (1991) show permissive results in the Nash equilibrium by considering stochastic SCFs and stochastic mechanism design, and then requiring not exact but virtual (i.e., approximate) implementability. Abreu and Matsushima (1992a) strengthens these results in virtual implementation by replacing Nash equilibrium with a much weaker solution concept termed iterative dominance, as well as by utilizing only bounded mechanisms (e.g., Abreu-Matsushima mechanisms). Palfrey and Srivastava (1991) replaces Nash equilibrium with a more restrictive solution concept termed weakly undominated Nash equilibrium, and then derived a possibility result in exact implementation. Abreu and Matsushima (1994) strengthens this result by replacing the uniqueness of weakly undominated Nash equilibrium with a more restrictive requirement, that is, the unique survival from the iterative elimination of weakly dominated strategies. Moore and Repullo (1988) derive a possibility result in exact implementation by replacing the Nash equilibrium with a refinement, that is, subgame perfect equilibrium. Matsushima (2008a, b) derive possibility results in exact implementation by considering behavioral aspects of players such as preferences for honesty.

Matsushima (2019) is the first work to incorporate ex-post verifiability into unique implementation, showing a very permissive result, such that with full ex-post verifiability, any SCF is uniquely and exactly implementable in iterative dominance ex-post. This is regarded as the most demanding of the solution concepts used in this literature. Matsushima (2019) imposes various severe restrictions such as boundedness, permission of only small transfers off the equilibrium path, and no permission of transfers on the equilibrium path. Moreover, Matsushima (2019) does not assume either expected utility or quasi-linearity.

Assuming the availability of ex-post verifiable information is plausible in realistic situations. By conducting a follow-up survey, the central planner can generally obtain a resultant verifiable consequence of an allocation decision. The importance of evaluating past policies afterwards and making use of this evaluation to improve future policy decisions is getting widely acknowledged. Matsushima (2019) aims to enhance incentives for players to coordinate with each other in achieving more desirable policies by utilizing subsequent evaluations as threats.

However, the argument in Matsushima (2019) relies on the full verifiability of the state. It is generally unrealistic to assume that the state is fully ex-post verifiable, even if the technology to properly process data will substantially improve in the future. This study aims to extend the permissive result in Matsushima (2019) to the more general case of partial ex-post verifiability. This extension is by no means an easy task: without full verifiability, in a simple majority rule, players can successfully coordinate to communicate the same lie about the unverifiable parts of the state. Hence, we must develop a different mechanism design to detect such lies, and then clarify a condition under which this design method functions. This study proposes a new method for mechanism design and shows that full detection is 
a sufficient condition to guarantee the same result as in Matsushima (2019), even if we replace full ex-post verifiability with partial ex-post verifiability (full detection).

This paper is organized as follows. Section 2 describes the basic model. Section 3 defines iterative dominance and unique implementation. Section 4 introduces full detection. Section 5 argues for the necessity of full detection. Section 6 shows the main theorem, and Sect. 7 concludes.

\section{The model}

We consider a situation in which the central planner determines an allocation and makes monetary transfers. Let $N \equiv\{1, \ldots, n\}$ denote the finite set of all players, where we assume $n \geq 3$. Let $A$ denote the finite set of all allocations. Let $\Delta$ denote the set of all lotteries over allocations. We denote $\alpha \in \Delta$. We write $\alpha=a$ if $\alpha(a)=1$. Let $\Omega$ denote the finite set of all states, that is, the state space. An SCF is defined as $f: \Omega \rightarrow \Delta .^{2}$ The state-contingent utility function for each player $i \in N$ is defined as:

$$
u_{i}: \Delta \times R \times \Omega \rightarrow R,
$$

where $u_{i}\left(\alpha, t_{i}, \omega\right)$ is the utility for player $i$ when the state $\omega$ occurs; the central planner determines an allocation according to the lottery $\alpha \in \Delta$, and he (or she) makes a monetary transfer $t_{i} \in R$ to player $i$. We assume that $u_{i}\left(\alpha, t_{i}, \omega\right)$ is continuous with respect to $\alpha \in \Delta$ and $t_{i} \in R$, and is increasing in $t_{i}$. We do not assume expected utility and quasi-linearity. ${ }^{3}$

Specifically, we describe a state as

$$
\omega=\left(\omega_{0}, \omega_{1}, \ldots, \omega_{n}\right) .
$$

For each $i \in N \cup\{0\}$, let $\Omega_{i}$ denote the set of possible $\omega_{i}$. We denote

$$
\omega_{-i} \equiv\left(\omega_{j}\right)_{j \in N \cup\{0\} \backslash\{i\}} \in \underset{j \in N \cup\{0\} \backslash\{i\}}{\times} \Omega_{j},
$$

and

$$
\omega_{-i-j} \equiv\left(\omega_{l}\right)_{l \in N \cup\{0\} \backslash\{i, j\}} \in \underset{l \in N \cup\{0\} \backslash\{i, j\}}{\times} \Omega_{l} .
$$

We assume that $\Omega$ is a proper subset of $\underset{i \in N \cup\{0\}}{\times} \Omega_{i}$, that is,

$$
\Omega \underset{\neq}{\subset} \underset{i \in N \cup\{0\}}{\times} \Omega_{i}
$$

Let $\Omega_{-i}\left(\omega_{i}\right) \subset \underset{j \in N \cup\{0\} \backslash\{i\}}{\times} \Omega_{j}$ denote the set of possible $\omega_{-i}$, such that

\footnotetext{
${ }^{2}$ Many works in the exact implementation literature investigated social choice correspondences instead of SCFs to soften the difficulty of full implementation. Jain and Lombardi (2009) investigated the virtual implementation of correspondences. This study does not investigate correspondences. I expect the extension of this study in this respect to be related to Jain and Lombardi, because both studies use stochastic mechanism design.
}

${ }^{3}$ See Matsushima (2019). 


$$
\left(\omega_{i}, \omega_{-i}\right) \in \Omega .
$$

We assume that $\Omega_{-i}\left(\omega_{i}\right)$ is nonempty for all $i \in N \cup\{0\}$ and all $\omega_{i} \in \Omega_{i}$. We denote

$$
\Omega_{-i} \equiv \underset{\omega_{i} \in \Omega_{i}}{\times} \Omega_{-i}\left(\omega_{i}\right) .
$$

We assume partial ex-post verifiability; after the central planner determines an allocation, but before he (or she) determines monetary transfers, only $\omega_{0}$ becomes publicly observable and verifiable. The remaining part of the state $\omega_{-0}$ is unverifiable throughout.

We consider the following two-stage procedure of players' information acquisition and revelation. At stage 1 (earlier stage), each player $i \in N$ observes $\omega_{i} \in \Omega_{i}$ as his (or her) private signal. The central planner then requires each player $i$ to make his (or her) first announcement $m_{i}^{1}$. At stage 2 (later stage), each player $i \in N$ observes the remaining part of the state $\omega_{-i} \in \Omega_{-i}\left(\omega_{i}\right)$. The central planner then requires each player $i$ to make his (or her) second announcement $m_{i}^{2}$. Hence, we assume incomplete information at stage 1 , but assume complete information at stage 2 . We also assume imperfect information, that is, each player cannot observe the other players' first and second announcements.

We define a dynamic mechanism as $\Gamma \equiv\left(M^{1}, M^{2}, g, x\right)$, where $M^{1} \equiv \underset{i \in N}{\times} M_{i}^{1}$, and $M_{i}^{1}$ denotes the set of possible first messages of player $i ; M^{2} \equiv \underset{i \in N}{\times} \underset{i \in N}{M_{i}^{2}}$, and $M_{i}^{2}$ denotes the set of possible second messages of player $i ; g: M^{1} \times M^{2} \rightarrow \Delta$ denotes the allocation rule; $x \equiv\left(x_{i}\right)_{i \in N}$ denotes the transfer rule; and $x_{i}: M^{1} \times M^{2} \times \Omega_{0} \rightarrow R$ denotes the transfer rule for player $i$. We assume that both $M_{i}^{1}$ and $M_{i}^{2}$ are finite.

Each player $i$ makes his (or her) first announcement $m_{i}^{1} \in M_{i}^{1}$ at stage 1 , and his (or her) second announcement $m_{i}^{2} \in M_{i}^{2}$ at stage 2 . The central planner then selects an allocation according to $g\left(m^{1}, m^{2}\right) \in \Delta$. After the central planner selects an allocation, $\omega_{0}$ becomes verifiable. In other words, there exists a dummy player (player 0 ) who always announces $\omega_{0}$ truthfully after the central planner selects the allocation. After $\omega_{0}$ becomes verifiable, the central planner receives the monetary transfer $x_{i}\left(m^{1}, m^{2}, \omega_{0}\right) \in R$ from each player $i$. Because of ex-post verifiability, the allocation rule $g$ does not depend on $\omega_{0}$, while the transfer rule $x$ does depend on $\omega_{0}$.

The strategy for player $i$ in a dynamic mechanism $\Gamma$ is defined as $s_{i} \equiv\left(s_{i}^{1}, s_{i}^{2}\right)$, where $s_{i}^{1}: \Omega_{i} \rightarrow M_{i}^{1}$ and $s_{i}^{2}: \Omega \rightarrow M_{i}^{2}$. According to $s_{i}$, at stage 1 , player $i$ announces $s_{i}^{1}\left(\omega_{i}\right) \in M_{i}^{1}$, which does not depend on $\omega_{-i}$. At stage 2 , he (or she) announces $s_{i}^{2}(\omega) \in M_{i}^{2}$, which does depend on $\omega_{-i}$ as well as $\omega_{i}$. Note from the imperfect information that each player's announcements do not depend on the other players' announcements. For each $t \in\{1,2\}$, let $S_{i}^{t}$ denotes the set of possible $s_{i}^{t}$. Let $S_{i} \equiv S_{i}^{1} \times S_{i}^{2}$ denote the set of all strategies for player $i$. Let $S^{t} \equiv \underset{i \in N}{\times} S_{i}^{t}, s^{t} \equiv\left(s_{i}^{t}\right)_{i \in N} \in S^{t}, S \equiv \underset{i \in N}{\times} S_{i}$, and $s \equiv\left(s_{i}\right)_{i \in N} \in S$. 


\section{Iterative dominance}

We define iterative dominance as the following ex-post term. For every $i \in N$, let

$$
M_{i}^{1}\left(0, \omega_{i}\right) \equiv M_{i}^{1} \quad \text { for all } \omega_{i} \in \Omega_{i},
$$

and

$$
M_{i}^{2}(0, \omega) \equiv M_{i}^{2} \quad \text { for all } \omega \in \Omega
$$

Let

$$
\begin{aligned}
& M^{1}\left(0, \omega_{-0}\right) \equiv \underset{i \in N}{\times} M_{i}^{1}\left(0, \omega_{i}\right), M_{-i}^{1}\left(0, \omega_{-0-i}\right) \equiv \underset{j \in N \backslash\{i\}}{\times} M_{j}^{1}\left(0, \omega_{j}\right), \\
& M^{2}(0, \omega) \equiv \underset{i \in N}{\times} M_{i}^{2}(0, \omega), \quad \text { and } M_{-i}^{2}(0, \omega) \equiv \underset{j \in N \backslash\{i\}}{\times} M_{j}^{2}(0, \omega) .
\end{aligned}
$$

We then iteratively remove messages that are dominated in the ex-post term; recursively, for each $h \geq 1$, we define $M_{i}^{1}\left(h, \omega_{i}\right) \subset M_{i}^{1}\left(h-1, \omega_{i}\right)$ in the manner that $m_{i}^{1} \in M_{i}^{1}\left(h, \omega_{i}\right)$ if and only if there is no $\tilde{m}_{i}^{1} \in M_{i}^{1}\left(h-1, \omega_{i}\right)$, such that for every $\omega_{-i} \in \Omega_{-i}\left(\omega_{i}\right), m_{-i}^{1} \in M_{-i}^{1}\left(h-1, \omega_{-0-i}\right)$, and $m^{2} \in M^{2}(h-1, \omega)$,

$$
\begin{aligned}
& u_{i}\left(g\left(m^{1}, m^{2}\right),-x_{i}\left(m^{1}, m^{2}, \omega_{0}\right), \omega\right) \\
& \quad \leq u_{i}\left(g\left(\tilde{m}_{i}^{1}, m_{-i}^{1}, m^{2}\right),-x_{i}\left(\tilde{m}_{i}^{1}, m_{-i}^{1}, m^{2}, \omega_{0}\right), \omega\right),
\end{aligned}
$$

and $\omega_{-i} \in \Omega_{-i}\left(\omega_{i}\right)$ exists, such that for every $m_{-i}^{1} \in M_{-i}^{1}\left(h-1, \omega_{-0-i}\right)$ and $m^{2} \in M^{2}(h-1, \omega)$,

$$
\begin{aligned}
& u_{i}\left(g\left(m^{1}, m^{2}\right),-x_{i}\left(m^{1}, m^{2}, \omega_{0}\right), \omega\right) \\
& \quad<u_{i}\left(g\left(\tilde{m}_{i}^{1}, m_{-i}^{1}, m^{2}\right),-x_{i}\left(\tilde{m}_{i}^{1}, m_{-i}^{1}, m^{2}, \omega_{0}\right), \omega\right) .
\end{aligned}
$$

Recursively, for each $h \geq 1$, we define $M_{i}^{2}(h, \omega) \subset M_{i}^{2}(h-1, \omega)$ in the manner that $m_{i}^{2} \in M_{i}^{2}(h, \omega)$ if and only if there is no $\tilde{m}_{i}^{2} \in M_{i}^{2}(h-1, \omega)$, such that for every $m_{-i}^{2} \in M_{-i}^{2}(h-1, \omega)$ and $m^{1} \in M^{1}\left(h-1, \omega_{-0}\right)$,

$$
\begin{aligned}
& u_{i}\left(g\left(m^{1}, m^{2}\right),-x_{i}\left(m^{1}, m^{2}, \omega_{0}\right), \omega\right) \\
& \quad<u_{i}\left(g\left(m^{1}, \tilde{m}_{i}^{2}, m_{-i}^{2}\right),-x_{i}\left(m^{1}, \tilde{m}_{i}^{2}, m_{-i}^{2}, \omega_{0}\right), \omega\right) .
\end{aligned}
$$

Let

$$
\begin{aligned}
& M^{1}\left(h, \omega_{-0}\right) \equiv \underset{i \in N}{\times} M_{i}^{1}\left(h, \omega_{i}\right), M_{-i}^{1}\left(h, \omega_{-0-i}\right) \equiv \underset{j \in N \backslash\{i\}}{\times} M_{j}^{1}\left(h, \omega_{j}\right), \\
& M^{2}(h, \omega) \equiv \underset{i \in N}{\times} M_{i}^{2}(h, \omega), \quad \text { and } M_{-i}^{2}(h, \omega) \equiv \underset{j \in N \backslash\{i\}}{\times} M_{j}^{2}(h, \omega) .
\end{aligned}
$$

We define 


$$
\begin{gathered}
M_{i}^{1}\left(\infty, \omega_{i}\right) \equiv \bigcap_{h=0}^{\infty} M_{i}^{1}\left(h, \omega_{i}\right), M^{1}\left(\infty, \omega_{-0}\right) \equiv \underset{i \in N}{\times} M_{i}^{1}\left(\infty, \omega_{i}\right), \\
M_{i}^{2}(\infty, \omega) \equiv \bigcap_{h=0}^{\infty} M_{i}^{2}(h, \omega), \quad \text { and } \quad M^{2}(\infty, \omega) \equiv \underset{i \in N}{\times} M_{i}^{2}(\infty, \omega) .
\end{gathered}
$$

Because of the finiteness, both $M_{i}^{1}\left(\infty, \omega_{i}\right)$ and $M_{i}^{2}(\infty, \omega)$ are non-empty, and there exists a positive integer $h^{*}$ such that for every $i \in N$ and $\omega \in \Omega$,

$$
M_{i}^{1}\left(h, \omega_{i}\right)=M_{i}^{1}\left(\infty, \omega_{i}\right) \quad \text { and } \quad M_{i}^{2}(h, \omega)=M_{i}^{2}(\infty, \omega) \quad \text { for all } h \geq h^{*} .
$$

Definition 3 A strategy $s_{i}=\left(s_{i}^{1}, s_{i}^{2}\right) \in S_{i}$ for player $i$ is said to be iteratively undominated in a dynamic mechanism $\Gamma$ if

$$
s_{i}^{1}\left(\omega_{i}\right) \in M_{i}^{1}\left(\infty, \omega_{i}\right) \quad \text { for all } \omega_{i} \in \Omega_{i},
$$

and

$$
s_{i}^{2}(\omega) \in M_{i}^{2}(\infty, \omega) \quad \text { for all } \omega \in \Omega .
$$

Definition 4 A dynamic mechanism $\Gamma$ is said to uniquely implement an SCF $f$ in iterative dominance if the unique iteratively undominated strategy profile $s \in S$ exists in $\Gamma$, and this profile induces the value of the $\mathrm{SCF}$, that is,

$$
g(s(\omega))=f(\omega) \text { for all } \omega \in \Omega .
$$

\section{Full detection}

This section demonstrates a condition on the state space $\Omega$, which we term full detection. For each $i \in N$, consider an arbitrary function $\chi_{-i}: \Omega_{-i} \rightarrow 2^{\Omega_{-i}}$, where we assume that for every $\omega_{-i} \in \Omega_{-i}$,

$$
\omega_{-i} \in \chi_{-i}\left(\omega_{-i}\right),
$$

and

$$
\tilde{\omega}_{0}=\omega_{0} \text { for all } \tilde{\omega}_{-i}=\left(\tilde{\omega}_{0}, \tilde{\omega}_{-0-i}\right) \in \chi_{-i}\left(\omega_{-i}\right) .
$$

The interpretation of $\chi_{-i}$ is as follows. Consider a direct revelation at stage 1. All players, including player 0 (the dummy player), are asked to announce their respective private signals. Here, player 0 is assumed to always announce $\omega_{0}$ truthfully. In this direct revelation, $\chi_{-i}$ describes a pattern of announcements by all players except for player $i$; they announce a profile that belongs to $\chi_{-i}\left(\omega_{-i}\right) \subset \Omega_{-i}$ whenever $\omega_{-i}$ occurs.

We introduce a notion on $\chi_{-i}$, termed detection, as follows. 
Definition 5 A function $\chi_{-i}$ is said to detect player $i$ for $\omega_{i}$ against $\omega_{i}^{\prime}$ if $\omega_{-i} \in \Omega_{-i}\left(\omega_{i}\right)$ exists, such that

$$
\chi_{-i}\left(\tilde{\omega}_{-i}\right) \cap \chi_{-i}\left(\omega_{-i}\right)=\phi \quad \text { for all } \tilde{\omega}_{-i} \in \Omega_{-i}\left(\omega_{i}^{\prime}\right) .
$$

Suppose that $\omega_{i}$ is correct, but player $i$ announces $\omega_{i}^{\prime} \neq \omega_{i}$. Suppose also that for every $\omega_{-i}$, the other players announce according to $\chi_{-i}\left(\omega_{-i}\right) \subset \Omega_{-i}$, that is, they announce a profile $\left(\omega_{0}, \omega_{-o-i}^{\prime}\right)$ that satisfies.

$$
\left(\omega_{0}, \omega_{-0-i}^{\prime}\right) \in \chi_{-i}\left(\omega_{-i}\right) .
$$

Note that if player $i^{\prime} s$ announcement $\omega_{i}^{\prime}$ is correct, the other players announce according to $\chi_{-i}\left(\tilde{\omega}_{-i}\right)$ for some $\tilde{\omega}_{-i} \in \Omega_{-i}\left(\omega_{i}^{\prime}\right)$, that is, they announce a profile $\left(\omega_{0}, \omega_{-0-i}^{\prime}\right)$ that satisfies

$$
\left(\omega_{0}, \omega_{-0-i}^{\prime}\right) \in \chi_{-i}\left(\tilde{\omega}_{-i}\right)
$$

Suppose that player $i$ expects $\omega_{-i}=\left(\omega_{0}, \omega_{-i-0}\right) \in \Omega_{-i}\left(\omega_{i}\right)$ to occur. Then, player $i$ will expect the other players to announce according to $\chi_{-i}\left(\omega_{-i}\right)$. However, the condition (1) implies that if player $i^{\prime} s$ announcement $\omega_{i}^{\prime}$ is correct, the other players never announce according to $\chi_{-i}\left(\omega_{-i}\right)$. This is a contradiction; in this case, we can recognize that player $i^{\prime} s$ announcement $\omega_{i}^{\prime}$ is incorrect. Hence, $\chi_{-i}$ successfully detects player $i$ for $\omega_{i}$ against $\omega_{i \cdot}^{\prime}{ }^{4}$

Based on this detection notion, we define full detection as the following condition on the state space $\Omega$. For every $h \in\{0,1, \ldots\}$, we specify $\chi_{i}(h): \Omega_{i} \rightarrow 2^{\Omega_{i}}$ for all $i \in N \cup\{0\}$, and $\chi_{-i}(h): \Omega_{-i} \rightarrow 2^{\Omega_{-i}}$ for all $i \in N$, in the following manner. Let

$$
\chi_{0}(0)\left(\omega_{0}\right)=\left\{\omega_{0}\right\} \text { for all } \omega_{0} \in \Omega_{0} .
$$

For every $i \in N$, let

$$
\chi_{i}(0)\left(\omega_{i}\right)=\Omega_{i}
$$

and

$$
\chi_{-i}(0)\left(\omega_{-i}\right)=\left\{\omega_{0}\right\} \times \Omega_{-i} .
$$

For each $h \in\{1,2, \ldots\}$, let

$$
\chi_{0}(h)\left(\omega_{0}\right)=\left\{\omega_{0}\right\} \quad \text { for all } \omega_{0} \in \Omega_{0} .
$$

Recursively, for every $h \in\{1,2, \ldots\}$ and $i \in N$, we define $\chi_{i}(h)\left(\omega_{i}\right) \subset \chi_{i}(h-1)\left(\omega_{i}\right)$ and $\chi_{-i}(h)\left(\omega_{-i}\right) \subset \chi_{-i}(h-1)\left(\omega_{-i}\right)$ in the following manner. For every $\omega_{i}^{\prime} \in \chi_{i}(h-1)\left(\omega_{i}\right)$

\footnotetext{
${ }^{4}$ Note that detection does not imply that a message is forbidden simply because it is different from the hard evidence. In this respect this study is different from Kartik and Tercieux (2012).
} 


$$
\begin{aligned}
\omega_{i}^{\prime} \in \chi_{i}(h)\left(\omega_{i}\right) \quad \text { if and only if } \chi_{-i}(h-1) \text { fails to detect player } i \\
\text { for } \omega_{i} \text { against } \omega_{i}^{\prime}, \text { that is, } \\
\left\{\begin{array}{c}
\left.U_{\tilde{\omega}_{-i} \in \Omega_{-i}\left(\omega_{i}^{\prime}\right)} \chi_{-i}(h-1)\left(\tilde{\omega}_{-i}\right)\right\} \cap \chi_{-i}(h-1)\left(\omega_{-i}\right) \neq \phi \text { for all } \\
\omega_{-i} \in \Omega_{-i}\left(\omega_{i}\right),
\end{array}\right.
\end{aligned}
$$

and for every $\omega_{-i}^{\prime} \in \chi_{-i}(h-1)\left(\omega_{-i}\right)$,

$$
\begin{aligned}
& \omega_{-i}^{\prime} \in \chi_{-i}(h)\left(\omega_{-i}\right) \text { if and only if } \omega_{j} \in \chi_{j}(h)\left(\tilde{\omega}_{j}\right) \text { for all } \\
& j \quad j \in N \cup\{0\} \backslash\{i\} .
\end{aligned}
$$

Note that $\chi_{-i}(h)$ implies the set of all announcements by all players except for player $i$ that can survive through the $h$-round iterative removal of detected lies. Importantly, $\chi_{i}(h)\left(\omega_{i}\right)$ is a partition of player $i^{\prime} s$ private signals, that is, for every $\omega_{i} \in \Omega_{i}$ and $\omega_{i}^{\prime} \in \Omega_{i}$, either $\chi_{i}(h)\left(\omega_{i}\right)=\chi_{i}(h)\left(\omega_{i}^{\prime}\right)$ or $\chi_{i}(h)\left(\omega_{i}\right) \cap \chi_{i}(h)\left(\omega_{i}^{\prime}\right)=\phi$.

We define

$$
\chi_{i}(\infty)\left(\omega_{i}\right) \equiv \bigcap_{h=0}^{\infty} \chi_{i}(h)\left(\omega_{i}\right)
$$

Since $\Omega$ is finite, it follows that $\chi_{i}(\infty)\left(\omega_{i}\right)$ is non-empty, and a positive integer $h^{* *}$ exists, such that

$$
\chi_{i}(h)\left(\omega_{i}\right)=\chi_{i}(\infty)\left(\omega_{i}\right) \quad \text { for all } h \geq h^{* *} .
$$

Full detection: The state space $\Omega$ is said to satisfy full detection if for every $i \in N$ and $\omega_{i} \in \Omega_{i}$,

$$
\chi_{i}(\infty)\left(\omega_{i}\right)=\left\{\omega_{i}\right\}
$$

Full detection implies that the iterative removal of detected lies eventually eliminates all lies; truth-telling is therefore the only announcement that survives through this removal procedure. Full detection assumes that there exists a very rare event, to which each player assigns a probability of occurrence of zero, and therefore he (or she) can ignore this event. As pointed out by the authors in behavioral economics such as Camerer and Kunreuther (1989), real people tend to assign a very rare event with probability zero because of their cognitive limitation. This justifies the relevancy of full detection.

To help the readers' understanding, let us consider the following two examples that satisfy full detection. These examples indicate that full detection is not restrictive in various interesting environments.

Example 1 (financial conditions) Consider $n+1$ companies, that is, company 0 , company $1, \ldots$, and company $n$. Each company $i^{\prime} s$ financial condition is described by $\omega_{i} \in \Omega_{i}$, where $\Omega_{i}=\Omega_{0}$ for all $i \in N$. We assume that the financial condition of company 0 (dummy player) can be verified ex-post. We assume that the financial condition of each company $i, \omega_{i} \in \Omega_{i}$ is not much different from its neighbor 
company $i-1^{\prime} s$ financial condition $\omega_{i-1}$. To be precise, there exists a one-to-one mapping $\rho: \Omega_{0} \rightarrow \Omega_{0}$ such that for every $\omega \in \underset{i \in N \cup\{0\}}{\times} \Omega_{i}$, $\omega \in \Omega$ if and only if $\omega_{i-1} \neq \rho\left(\omega_{i}\right)$ for all $i \in N$.

Here, we interpret $\rho\left(\omega_{i}\right)$ as the opposite extreme of the financial condition $\omega_{i}$. Hence, each company's financial condition cannot be the opposite extreme of its neighbor company.

The ex-post verified information $\omega_{0}$ can directly detect any lie about $\omega_{1}$, because player 1 cannot exclude the possibility that his lie $\omega_{1}^{\prime} \neq \omega_{1}$ is equivalent to $\rho\left(\omega_{0}\right)$. This motivates player 1 to tell the truth about $\omega_{1}$. In the same manner, the truthful announcement about $\omega_{1}$ can detect any lie about $\omega_{2}$. This motivates player 2 to tell the truth about $\omega_{2}$. Recursively, any player $i \in\{1, \ldots, n\}$ is well motivated to tell the truth about $\omega_{i}$, implying full detection.

Example 2 (Earthquake) Consider the government (dummy player 0) that attempts to know whether the earthquake occurs in a nuclear plant belt. The government receives information, which is either $\mathrm{Y}, \mathrm{N}$, or U. Here, "Y" implies that the earthquake occurs, "N" implies that the earthquake never occurs, and " $U$ " implies no information about the occurrence of the earthquake. There are $n$ experts, each of whom receives his (or her) respective information that is either $\mathrm{Y}, \mathrm{N}$, or $\mathrm{U}$. We assume that if an expert or the government receives $\mathrm{Y}(\mathrm{N})$, then all other experts and the government never receives $\mathrm{N}$ (Y, respectively). We also assume that whenever the government fails to know about the occurrence of the earthquake, i.e., receives $\mathrm{U}$, then there exists at least one expert who know about the occurrence of the earthquake, i.e., receives either $\mathrm{Y}$ or $\mathrm{N}$. To be precise, this example is described as follows:

$$
\Omega_{i}=\{Y, N, U\} \text { for all } i \in N \cup\{0\},
$$

and $\omega \in \Omega$ if and only if

$$
\omega_{i} \neq U \text { for some } i \in N \cup\{0\},
$$

and for every $i \in N \cup\{0\}$ and $j \in N \cup\{0\}$,

$$
\omega_{i}=\omega_{j} \text { whenever } \omega_{i} \neq U \text { and } \omega_{j} \neq U
$$

We can show that this example satisfies full detection as follows. Each expert $i \in N$ never announces $m_{i} \notin\left\{U, \omega_{i}\right\}$ whenever $\omega_{i} \neq U$, because it might be the case that $\omega_{0}=\omega_{i}$, which detects his (or her) lie. Each expert $i \in N$ never announces $m_{i} \neq U$ whenever $\omega_{i}=U$, because it might be the case that $\omega_{0} \notin\left\{U, m_{i}\right\}$, which detects his (or her) lie. From these observations, it follows that each expert $i \in N$ never announces $m_{i}=U$ whenever $\omega_{i} \neq U$, because it might be the case that $\omega_{j}=U$ for all $j \in N \cup\{0\} \backslash\{i\}$, which implies $\omega_{i} \neq U$, detecting his (or her) lie. 


\section{Necessity of full detection}

To understand the role of full detection, this section considers a special case where we assume that each player does not concern the allocation selection but only concerns the resultant transfer. (We will remove this assumption from the next section.) We then investigate a hypothetical direct revelation in which, at stage 1, each player $i \in N$ announces his (or her) private signal $\omega_{i}$, and after $\omega_{0}$ becomes observable and verifiable, the central planner makes transfers according to a transfer rule $x$. This corresponds to a degenerate case of dynamic mechanism, termed a direct revelation mechanism. Here, for every player $i \in N$, his (or her) second message space $M_{i}^{2}$ is a singleton, $M_{i}^{1}=\Omega_{i}$, and

$$
u_{i}\left(\alpha, t_{i}, \omega\right)=t_{i} \quad \text { for all } \alpha \in \Delta \quad \text { and } \quad t_{i} \in R .
$$

Hence, we can simply write $x$ for a dynamic mechanism $\Gamma=\left(M^{1}, M^{2}, g, x\right)$, denote $x_{i}\left(m^{1}, \omega_{0}\right)$ instead of $x_{i}\left(m^{1}, m^{2}, \omega_{0}\right)$, and denote $s_{i}^{1}$ instead of $s_{i}=\left(s_{i}^{1}, s_{i}^{2}\right)$. Without loss of generality, we can focus on the possibility that the honest strategy profile $s^{1 *}=\left(s_{i}^{1 *}\right)_{i \in N}$ is the unique iteratively undominated strategy profile in the direct revelation, where

$$
s_{i}^{1 *}\left(\omega_{i}\right)=\omega_{i} \quad \text { for all } i \in N \quad \text { and } \quad \omega_{i} \in \Omega_{i} .
$$

For every $h \in\left\{1, \ldots, h^{*}\right\}$, we fix an arbitrary positive real number $\eta_{1}(h)>0$. We assume that

$$
\eta_{1}(\tilde{h})>\sum_{h=\tilde{h}+1}^{h^{*}} \eta_{1}(h) \quad \text { for all } \tilde{h} \in\left\{1, \ldots, h^{*}\right\} .
$$

We specify a transfer rule $x^{*}=\left(x_{i}^{*}\right)_{i \in N}$ as follows. For every $i \in N, m^{1} \in M^{1}$, and $\omega_{0} \in \Omega_{0}$,

$$
x_{i}^{*}\left(m^{1}, \omega_{0}\right)=\sum_{h=1}^{h^{*}} x_{i}^{h}\left(m^{1}, \omega_{0}\right),
$$

where, for each $h \in\left\{1, \ldots, h^{*}\right\}$,

$$
x_{i}^{h}\left(m^{1}, \omega_{0}\right)=\eta_{1}(h) \text { if }\left(\omega_{0}, m_{-i}^{1}\right) \notin \underset{\tilde{\omega}_{-i} \in \Omega_{-i}\left(m_{i}^{1}\right)}{\cup} \chi_{-i}(h-1)\left(\tilde{\omega}_{-i}\right),
$$

and

$$
x_{i}^{h}\left(m^{1}, \omega_{0}\right)=0 \text { if }\left(\omega_{0}, m_{-i}^{1}\right) \in \underset{\tilde{\omega}_{-i} \in \Omega_{-i}\left(m_{i}^{1}\right)}{\cup} \chi_{-i}(h-1)\left(\tilde{\omega}_{-i}\right)
$$

According to $x_{i}^{*}$, any player $i \in N$ is fined the monetary amount $\eta_{1}(h)$ if he (or she) makes an announcement that is detected by $\chi_{-i}(h-1)$, which describes the profiles of the other players' announcements survived through the $(h-1)-$ round iterative removals of detected messages. This holds true irrespective of $h$. 
From (2), it follows that, with full detection, each player is willing to announce an undetected message, that is, the honest message.

Theorem 1 Consider the direct revelation. If the state space satisfies full detection, then the honest strategy profile $s^{1 *}$ is the unique iteratively undominated strategy profile in the specified direct revelation mechanism $x^{*}$. If a direct revelation mechanism $x$ exists in which $s^{1 *}$ is the unique iteratively undominated strategy profile, then the state space satisfies full detection.

Proof The proof of the first half of this theorem is as follows. Suppose that player $i$ observes $\omega_{i}$ and announces $m_{i}^{1} \notin \chi_{i}(1)\left(\omega_{i}\right)$. In this case, $\chi_{-i}(0)$ detects him (or her) for $\omega_{i}$ against $m_{i}^{1} ; \omega_{-i} \in \Omega_{-i}\left(\omega_{i}\right)$ exists, such that

$$
\chi_{-i}(0)\left(\tilde{\omega}_{-i}\right) \cap \chi_{-i}(0)\left(\omega_{-i}\right)=\phi \quad \text { for all } \tilde{\omega}_{-i} \in \Omega_{-i}\left(m_{i}^{1}\right) .
$$

Since $\chi_{-i}(0)\left(\omega_{-i}\right)=\left\{\omega_{0}\right\} \times M_{-0-i}^{1}$, the announcement by any other player $j \in N \backslash\{i\}$ belongs to $\chi_{j}(0)\left(\omega_{j}\right)$. This implies that, by announcing $m_{i}^{1}$, he (or she) is fined $\eta_{1}(1)$. In contrast, he (or she) can avoid this fine by announcing $\omega_{i}$ truthfully. Since $\eta_{1}(1)$ is large enough to satisfy (2), player $i$ never announces any element that does not belong to $\chi_{i}(1)\left(\omega_{i}\right)$.

Consider an arbitrary $h \in\left\{2, \ldots, h^{* *}\right\}$. Suppose that any player $i \in N$ announces a message that belongs to $\chi_{i}(h-1)\left(\omega_{i}\right)$. Suppose that player $i$ observes $\omega_{i}$ and announces $m_{i}^{1} \notin \chi_{i}(h)\left(\omega_{i}\right)$. In this case, $\chi_{-i}(h-1)$ detects him (or her) for $\omega_{i}$ against $m_{i}^{1}$. That is, $\omega_{-i} \in \Omega_{-i}\left(\omega_{i}\right)$ exists, such that

$$
\chi_{-i}(h-1)\left(\tilde{\omega}_{-i}\right) \cap \chi_{-i}(h-1)\left(\omega_{-i}\right)=\phi \quad \text { for all } \tilde{\omega}_{-i} \in \Omega_{-i}\left(m_{i}^{1}\right) .
$$

Since the announcement by any other player $j \in N \backslash\{i\}$ belongs to $\chi_{j}(h-1)\left(\omega_{j}\right)$, he (or she) is fined $\eta_{1}(h)$. In contrast, he (or she) can avoid this fine by announcing $\omega_{i}$ truthfully. Since $\eta_{1}(h)$ is large enough to satisfy (2), player $i$ never announces any element that does not belong to $\chi_{i}(h)\left(\omega_{i}\right)$.

From the above arguments, we have proved that if $s_{i}^{1}$ is strictly iteratively undominated, then

$$
s_{i}^{1}\left(\omega_{i}\right) \in \bigcap_{h \rightarrow \infty} \chi_{i}(h)\left(\omega_{i}\right) \text { for all } \omega_{i} \in \Omega_{i},
$$

which, along with full detection, implies that

$$
s_{i}^{1}\left(\omega_{i}\right)=\omega_{i} \text { for all } \omega_{i} \in \Omega_{i}, \quad \text { that is, } s_{i}^{1}=s_{i}^{1 *} .
$$

The proof of the second half of this theorem is as follows. Consider an arbitrary direct revelation mechanism $x$, and suppose $s^{1 *}$ is the unique iteratively undominated strategy profile in $x$. Note that

$$
u_{i}\left(g\left(m^{1}, m^{2}\right),-x_{i}\left(m^{1}, m^{2}, \omega_{0}\right), \omega\right)=-x_{i}\left(m^{1}, \omega_{0}\right),
$$

which implies that each player $i^{\prime} s$ incentive relies only on $\left(m_{i}, \omega_{0}\right)$. This, along with the definitions of iterative dominance and $\chi_{i}(1)\left(\omega_{i}\right)$, automatically implies that 


$$
M_{i}^{1}\left(1, \omega_{i}\right)=\chi_{i}(1)\left(\omega_{i}\right)
$$

We fix $h \geq 2$ arbitrarily, and suppose that

$$
M_{i}^{1}\left(h-1, \omega_{i}\right)=\chi_{i}(h-1)\left(\omega_{i}\right) \quad \text { for all } i \in N \quad \text { and } \quad \omega_{i} \in \Omega_{i} .
$$

Since each player $i^{\prime} s$ incentive relies only on $\left(m_{i}, \omega_{0}\right)$, in the same manner as above, it follows from the definitions of iterative dominance and $\chi_{i}(1)\left(\omega_{i}\right)$ that

$$
M_{i}^{1}\left(h, \omega_{i}\right)=\chi_{i}(h)\left(\omega_{i}\right) .
$$

Hence, we have proved that if $s_{i}^{1 *}$ is strictly iteratively undominated, then

$$
M_{i}^{1}\left(\infty, \omega_{i}\right)=\chi_{i}(\infty)\left(\omega_{i}\right)=\left\{\omega_{i}\right\},
$$

implying full detection.

\section{Unique implementation}

Theorem 1 implies that full detection is necessary and sufficient for unique implementation on the assumption that each player only concerns the resultant monetary transfer to him (or her). This section will remove this assumption and shows that full detection is generally sufficient for unique implementation even if each player concerns the allocation selection as well as the monetary transfer.

To uniquely implement an SCF $f$ in iterative dominance, we consider the following manner of designing a dynamic mechanism instead of considering direct revelation mechanisms. We first fix the arbitrary positive real numbers,

$$
\eta_{1}(h)>0 \quad \text { for each } h \in\left\{1, \ldots, h^{*}\right\}, \eta_{2}>0, \quad \text { and } \quad \eta_{3}>0,
$$

where $\eta_{1}(h)$ was introduced in Sect. 5. We denote $\eta_{1} \equiv\left(\eta_{1}(h)\right)_{h=1}^{h^{*}}$ and assume the inequalities (2). We then fix an arbitrary integer $K>1$ and an arbitrary allocation $a^{*} \in A$.

According to the basic method explored by Abreu and Matsushima (1992a, b, 1994), we construct a dynamic mechanism $\Gamma^{*}=\Gamma^{*}\left(f, \eta_{1}, \eta_{2}, \eta_{3}, K, a^{*}\right)=\left(M^{1}, M^{2}, g, x\right)$ as follows. Let

$$
\begin{gathered}
M_{i}^{1}=\Omega_{i}, \\
M_{i}^{2}=\prod_{k=1}^{K} M_{i}^{2, k},
\end{gathered}
$$

and

$$
M_{i}^{2, k}=\Omega \quad \text { for all } k \in\{1, \ldots, K\}
$$


At stage 1, each player announces his (or her) private signal. Importantly, at stage 2 , each player announces the state $K$ times. We thus denote

$$
M^{2, k} \equiv \underset{i \in N}{\times} M_{i}^{2, k}
$$

For each $k \in\{1, \ldots, K\}$, we define $g^{k}: M^{2, k} \rightarrow \Delta$, such that for each $\omega \in \Omega$,

$$
g^{k}\left(m^{2, k}\right)=f(\omega) \text { if } m_{i}^{2, k}=\omega \text { for at least } n-1 \text { players, }
$$

and

$$
g^{k}\left(m^{2, k}\right)=a^{*} \text { if no such } \omega \text { exists. }
$$

We then specify

$$
g\left(m^{1}, m^{2}\right)=\frac{\sum_{k=1}^{K} g^{k}\left(m^{2, k}\right)}{K} .
$$

The allocation rule $g$ is independent of the first announcements $m^{1}$. The central planner randomly picks up an integer $k$ from 1 to $\mathrm{K}$, and using $g^{k}$ and $m^{2, k}$, determines an allocation according to the lottery $g^{k}\left(m^{2, k}\right)$.

Moreover, we specify

$$
x_{i}\left(m^{1}, m^{2}, \omega_{0}\right)=x_{i}^{*}\left(m^{1}, \omega_{0}\right)+z_{i}\left(m^{1}, m^{2}, \omega_{0}\right),
$$

where $x_{i}^{*}$ was defined in Sect. 5,

$$
\begin{aligned}
z_{i}\left(m^{1}, m^{2}, \omega_{0}\right)= & \eta_{2}+\frac{r_{i}}{K} \eta_{3} \text { if } k \in\{1, \ldots, K\} \text { exists, such that } \\
& m_{i}^{2, k} \neq\left(\omega_{0}, m^{1}\right), \quad \text { and } \\
& m_{j}^{2, k^{\prime}}=\left(\omega_{0}, m^{1}\right) \quad \text { for all } k^{\prime}<k \text { and } \\
& j \in N \backslash\{i\},
\end{aligned}
$$

and

$$
z_{i}\left(m^{1}, m^{2}, \omega_{0}\right)=\frac{r_{i}}{K} \eta_{3} \text { if no such } k \in\{1, \ldots, K\} \text { exists. }
$$

Here, $r_{i} \in\{0, \ldots, K\}$ denotes the number of $k \in\{2, \ldots, K\}$, such that $m_{i}^{2, k} \neq\left(\omega_{0}, m^{1}\right)$.

In addition to (2), we assume for $\left(\eta_{1}, \eta_{2}, \eta_{3}\right)$ that

$$
\eta_{1}(\tilde{h})>\sum_{h=\tilde{h}+1}^{h^{*}} \eta_{1}(h)+\eta_{2}+\eta_{3} \quad \text { for all } \tilde{h} \in\left\{1, \ldots, h^{*}\right\} .
$$

From the specification of $x$, for every $i \in N$ and $\left(m^{1}, m^{2}, \omega_{0}\right) \in M^{1} \times M^{2} \times \Omega_{0}$, 


$$
0 \leq x_{i}\left(m^{1}, m^{2}, \omega_{0}\right) \leq \sum_{h=1}^{h^{*}} \eta_{1}(h)+\eta_{2}+\eta_{3} .
$$

By choosing $\sum_{h=1}^{h^{*}} \eta_{1}(h)+\eta_{2}+\eta_{3}$ as close to zero as possible, we can make the monetary transfer $x_{i}\left(m^{1}, m^{2}, \omega_{0}\right)$ as close to zero as possible.

Because of continuity, we can select a sufficiently large $K$, such that whenever

$$
\max _{a \in A}\left|\alpha(a)-\alpha^{\prime}(a)\right| \leq \frac{1}{K}
$$

then

$u_{i}\left(\alpha,-t_{i}, \omega_{i}\right)>u_{i}\left(\alpha^{\prime},-t_{i}-\eta_{2}, \omega_{i}\right) \quad$ for all $t_{i} \in\left[0, \sum_{h=1}^{h^{*}} \eta_{1}(h)+\eta_{3}\right] \quad$ and $\quad \omega_{i} \in \Omega_{i}$.

The inequalities (5) imply that $\eta_{2}$ is close to zero but is sufficiently large compared with the change of allocation within the $\frac{1}{K}-$ limit.

We define the honest strategy for player $i$ in $\Gamma^{*}$, denoted by $s_{i}^{*}=\left(s_{i}^{1 *}, s_{i}^{2 *}\right)$, as

$$
s_{i}^{1 *}\left(\omega_{i}\right)=\omega_{i} \text { for all } \omega_{i} \in \Omega_{i},
$$

and

$$
s_{i}^{2, k *}(\omega)=\omega \text { for all } k \in\{1, \ldots, K\} \text { and } \omega \in \Omega,
$$

where we denote $s_{i}^{2 *}=\left(s_{i}^{2, k *}\right)_{k=1}^{K}$ and $s_{i}^{2, k *}: \Omega \rightarrow \Omega$. The honest strategy profile $s^{*} \equiv\left(s_{i}^{*}\right)_{i \in N}$ induces the value of the SCF $f$ in $\Gamma^{*}$, that is,

$$
g\left(s^{*}(\omega)\right)=f(\omega) \text { for all } \omega \in \Omega .
$$

Note also that $s^{*}$ induces no monetary transfers on the equilibrium path, that is,

$$
x_{i}\left(s^{*}(\omega), \omega\right)=0 \quad \text { for all } i \in N \quad \text { and } \quad \omega \in \Omega .
$$

Theorem 2 Suppose that the state space satisfies full detection. Then, the honest strategy profile $s^{*}$ is the unique iteratively undominated strategy profile in $\Gamma^{*}$.

Proof Since the announcement of $m_{i}^{1}$ is irrelevant to the allocation choice and $\eta_{1}$ satisfies (2) and (3), we can prove, in the same manner as Theorem 1, that if $s_{i}$ is strictly iteratively undominated in $\Gamma^{*}$, then

$$
s^{1}=s^{1 *} \text {. }
$$

We show below that each player $i \in N$ prefers $m_{i}^{2,1}=m^{1}=\omega$. Suppose that another player $j \in N \backslash\{i\}$ exists who announces $m_{j}^{2,1} \neq \omega$. In this case, by announcing $m_{i}^{2,1} \neq \omega$ instead of $\omega$, player $i$ is fined $\eta_{2}$ or even more, while the resultant change of allocation is within the limit implied by (4). Hence, from (5), 
the impact of the fine $\eta_{2}$ on his (or her) welfare is greater than the impact of the resultant change of allocation.

Next, suppose that no player $j \in N \backslash\{i\}$ exists other than player $i$ who announces $m_{j}^{2,1} \neq \omega$. Then, by announcing $m_{i}^{2,1} \neq \omega$ instead of $\omega$, player $i$ is fined $\frac{\eta_{3}}{K}$ or even more (Note that even if he [or she] announces $m_{i}^{2,1}=\omega$, he [or she] may be one of the first deviants, and therefore, he [or she] does not necessarily avoid the fine $\eta_{2}$ ). From the specification of $g$ and $n \geq 3$, there is no resultant change of allocation. From these observations, he (or she) prefers $m_{i}^{2,1}=\omega$ regardless of the other players' announcements.

We now fix an arbitrary integer $h \in\{2, \ldots, K\}$. Suppose that any player $i \in N$ announces $m_{i}^{2, h^{\prime}}=\omega$ for all $h^{\prime} \in\{1, \ldots, h-1\}$. Suppose that a player $j \in N \backslash\{i\}$ exists other than player $i$ who announces $m_{j}^{2, h} \neq \omega$. Then, by announcing $m_{i}^{2, h} \neq \omega$ instead of $\omega$, player $i$ is fined $\eta_{2}$ or even more. In the same manner as above, the impact of the fine $\eta_{2}$ on his (or her) welfare is greater than the impact of the resultant change of allocation. Next, suppose that no player $j \in N \backslash\{i\}$ exists other than player $i$ who announces $m_{j}^{2, h} \neq \omega$. Then, by announcing $m_{i}^{2, h} \neq \omega$ instead of $\omega$, player $i$ is fined $\frac{\eta_{3}}{K}$ or even more. In the same manner as above, there is no resultant change of allocation in this case. Hence, he (or she) prefers $m_{i}^{2, h}=\omega$.

From these arguments, we have proved that $s_{i}^{2}=s_{i}^{2 *}$. Hence, we have completed the proof of this theorem.

Following the same logic as Theorem 1, we can prove that players' truth-telling at stage 1 is the unique equilibrium behavior. By using their truthful revelations at stage 1 and the ex-post verifiable information as the reference, we apply the same logic as Abreu and Matsushima (1992a, b, 1994) and Matsushima (2019) to prove that players are willing to make a truthful profile of announcements at stage 2 . Abreu and Matsushima (1992a) demonstrated a method of bounded (i.e., plausible) mechanism design, that is, Abreu-Matsushima mechanism, according to which, each player makes multiple announcements under imperfect information and the first deviant from the reference is fined.

According to $z_{i}$, any first deviant from the combination of the profile of first announcements and the ex-post verified information $\left(\omega^{0}, m_{0}\right)$ at stage 2 is fined the monetary amount $\eta_{2}$. Any player is additionally fined $\frac{\eta_{3}}{K}$ whenever he (or she) deviates from $\left(m^{1}, \omega_{0}\right)$. By setting the announcements at stage 1 and the ex-post verified information as the reference, any player is incentivized to make truthful announcements at stage 2 as unique equilibrium behavior. ${ }^{5}$

From Theorem 2, we conclude that with full detection, any SCF is uniquely implementable in iterative dominance, where we design a bounded mechanism, use no monetary transfers on the equilibrium path, and use only tiny monetary transfers even off the equilibrium path.

\footnotetext{
5 There are other methods of mechanism design such that modulo construction and integer game in the full (unique) implementation literature. In contrast with Abreu-Matsushima mechanism, these methods are far from satisfaction from the practical viewpoint. See Osborne and Rubinstein (1994, Chapter 10), for instance.
} 
Remark 1 This study assumes complete information at stage 2. However, we can eliminate this assumption without substantial changes by assuming ex-post incentive compatibility additionally. Hence, we can derive the same results as Theorems 1 and 2 , even if players receive no additional information at stage $2 .{ }^{6}$ We can extend this study to a case in which each player receives only additional information at stage 2 as an element of a partition of the state space. We can also derive the same results for the two-player case.

Remark 2 It is generally impossible for an SCF to be uniquely implementable in iterative dominance if the state space has the full support, that is, $\Omega=\underset{i \in N \cup\{0\}}{\times} \Omega_{i}$. On the other hand, by considering a signal-contingent probability function on $\Omega_{0}$, that is, $p_{i}\left(\omega_{i}\right): \Omega_{0} \rightarrow[0,1]$, we can derive possibility results similar to Theorems 1 and 2 , where we assume that for every $i \in N, \omega_{i} \in \Omega_{i}$, and $\omega_{i}^{\prime} \in \Omega_{i} \backslash\left\{\omega_{i}\right\}$,

$$
p_{i}\left(\omega_{i}\right) \neq p_{i}\left(\omega_{i}^{\prime}\right)
$$

and utilize a device of scoring rules. However, in this case, it is generally impossible for a transfer rule to satisfy zero monetary transfers on the equilibrium path.

\section{Concluding remarks}

This study investigated the unique implementation of an SCF in iterative dominance in the ex-post term. We assumed partial ex-post verifiability; that is, after determining an allocation, the central planner can observe partial but verifiable information about the state and make ex-post monetary transfers contingent on this information. We demonstrated a condition on the state space (full detection), which we prove is a sufficient condition for unique implementation. We constructed a dynamic mechanism with boundedness, in which each player announces his (or her) private signal at stage 1 before the other players observe it. In this construction, we only used small monetary transfers off the equilibrium path, but no transfers on the equilibrium path.

Our study contributes to the field of dynamic mechanism design (e.g., Krähmer and Strausz 2015). Penta (2015) investigates full implementation in incomplete information environments, where players receive information over time. Unlike this study, the author does not consider verifiability and adopts interim solution concepts such as perfect Bayesian equilibrium instead of ex-post-term concepts. Hence, it is important for future research to investigate a more dynamic situation than this study considered, in which stage 1 is divided into multiple sub-stages through which each player gradually acquires the whole picture of his (or her) private signals. Here, the

\footnotetext{
${ }^{6}$ Due to partial ex-post verifiability and full detection, we do not need any additional assumption such as measurability (Abreu and Matsushima 1992b) for unique implementation.
} 
central planner requires each player to make an announcement at each sub-stage. By considering these sub-stages, we expect to demonstrate weaker sufficient conditions than full detection.

This study is related to the works by Kartik and Tercieux (2012) and Ben-Porath and Lipman (2012), which investigate full implementation with hard evidence. These works assume that each player can receive verifiable information at early stages as hard evidence, and state that the degree to which hard evidence in the ex-ante and interim terms directly proves players' announcements to be correct is crucial in implementing a wide variety of SCFs. In contrast, this study emphasizes that a wide variety of SCFs are implementable even if the information is only verifiable ex-post and to a limited extent. To deepen our understanding of the role of verifiability in implementation, future research could explore integrating our study with these works.

Open Access This article is licensed under a Creative Commons Attribution 4.0 International License, which permits use, sharing, adaptation, distribution and reproduction in any medium or format, as long as you give appropriate credit to the original author(s) and the source, provide a link to the Creative Commons licence, and indicate if changes were made. The images or other third party material in this article are included in the article's Creative Commons licence, unless indicated otherwise in a credit line to the material. If material is not included in the article's Creative Commons licence and your intended use is not permitted by statutory regulation or exceeds the permitted use, you will need to obtain permission directly from the copyright holder. To view a copy of this licence, visit http://creativeco mmons.org/licenses/by/4.0/.

\section{References}

Abreu D, Matsushima H (1992a) Virtual implementation in iteratively undominated strategies: complete information. Econometrica 60:993-1008

Abreu D, Matsushima H (1992b): Virtual implementation in iteratively undominated strategies: incomplete information. Discussion Paper e-91-1, Hoover Institution, Stanford University

Abreu D, Matsushima H (1994) Exact implementation. J Econ Theory 64(1):1-19

Abreu D, Sen A (1991) Virtual implementation in Nash equilibrium. Econometrica 59:997-1021

Ben-Porath E, Lipman B (2012) Implementation with partial provability. J Econ Theory 147:1689-1724

Camerer C, Kunreuther H (1989) Decision processes for low probability events: policy implications. J Policy Anal Manag 8:565-592

Jackson M (1992) Implementation in undominated strategies: a look at bounded mechanisms. Rev Econ Stud 59:757-775

Jackson M (2001) A crash course in implementation theory. Soc Choice Welf 18:655-708

Jain R, Lombardi M (2009) Virtual implementation by bounded mechanisms: complete information. Institute of Academia Sinica, Taipei

Kartik N, Tercieux O (2012) Implementation with evidence. Theor Econ 7:323-356

Krähmer D, Strausz R (2015) Dynamic Mechanism Design. In: Börgers T (ed) An introduction to the theory of mechanism design. Oxford University Press, Oxford

Maskin E (1999) Nash equilibrium and welfare optimality. Rev Econ Stud 66:23-38

Maskin E, Sjöström T (2002) Implementation theory. In: Arrow K, Sen A, Suzumura K (eds) Handbook of social choice and welfare 1, vol 1. Elsevier, Amsterdam

Matsushima H (1988) A new approach to the implementation problem. J Econ Theory 45:128-144

Matsushima H (2008a) Role of honesty in full implementation. J Econ Theory 127:353-359

Matsushima H (2008b) Behavioral aspects of implementation theory. Econ Lett 100:161-164

Matsushima H (2017) Dynamic implementation, verification, and detection. CARF-F-416, University of Tokyo 
Matsushima H (2019) Implementation without expected utility: ex-post verifiability. Forthcoming in Social Choice and Welfare

Moore J (1992) Implementation in environments with complete information. In: Laffont JJ (ed) Advances in economic theory: sixth world congress. Cambridge University Press, Cambridge

Moore J, Repullo R (1988) Subgame perfect implementation. Econometrica 56:1191-1220

Osborne M, Rubinstein A (1994) A course in game theory. MIT Press, Cambridge

Palfrey T (1992) Implementation in Bayesian equilibrium: the multiple equilibrium problem in mechanism design. In: Laffont JJ (ed) Advances in economic theory: sixth world congress. Cambridge University Press, Cambridge

Palfrey T, Srivastava S (1991) Nash implementation using undominated strategies. Econometrica 59:479-501

Penta A (2015) Robust dynamic implementation. J Econ Theory 160:280-316

Publisher's Note Springer Nature remains neutral with regard to jurisdictional claims in published maps and institutional affiliations. 